\title{
Unravelling atherosclerosis
}

\section{James Scott}

AN Elevated level of blood cholesterol is a prerequisite for the development of atherosclerosis, the disease that causes heart attacks and strokes. Cholesterol is transported in the blood in low-density lipoprotein (LDL) particles. The rate of endocytosis of these particles by liver cells determines the level of blood cholesterol. This process is largely mediated by the LDL receptor and its two ligands apolipoprotein (apo)-B100 and apo-E. A full explanation of lipoprotein metabolism in people with familial hypercholesterolaemia caused by defects of the LDL receptor gene, however, requires the existence of another lipoprotein receptor specific for apo- $E^{1}$. Until now, the apo-E receptor has eluded purification and its existence has been questioned. With the publication by Herz et al. ${ }^{2}$ of the structure of a protein with remarkable resemblance to the LDL receptor, this controversy may have been resolved.

Another receptor of importance in the atherogenic process and cholesterol metabolism, the macrophage scavenger receptor, has also recently been isolated ${ }^{3.4}$. It mediates the clearance of oxidatively damaged LDL by macrophages - and may hold the key to atherogenesis. These discoveries should result in an increased understanding of the structural interaction between apolipoproteins and their receptors; the regulation of these receptors and their role in growth, development, inflammation and atherogenesis;

\section{0 years ago}

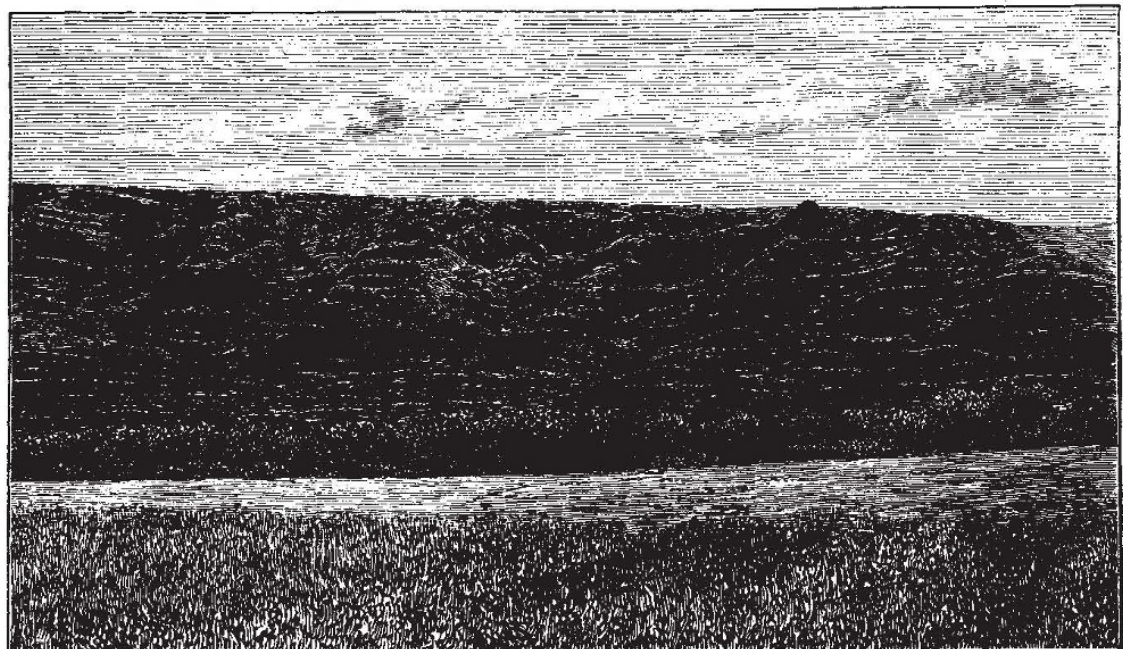

Mr. Ernst draws attention to the formation of these ledges as observed by himself in Caracas. They are probably to be found in many places, if carefully looked for. The diagram, taken from a photograph, illustrates one of the most striking instances I know, to be found near Ballantrae, on the Ballantrae-Girvan road, Ayrshire. The ledges occur on the western face of a series of low hills, ncar to the sea-shore. and the evolutionary relationship between the blood clotting, complement and lipoprotein systems and the molecular cascades that act during development.

Lipoproteins consist of a hydrophobic core of triglyceride and cholesteryl-ester surrounded by a polar coat of phospholipid and free cholesterol. The surface is stabilized by specific proteins called apolipoproteins. The largest of these, apo$\mathrm{B} 100$, is required for the secretion of very low-density lipoprotein (VLDL) by the liver. VLDL triglyceride is released by lipoprotein lipase in the blood capillaries, thereby converting VLDL to cholesterolrich LDL. Apo-B100 is the sole protein of LDL and the ligand that delivers cholesterol to cells by the LDL receptor. Intestinally derived lipoproteins called chylomicrons are assembled around apo-B48, which lacks the carboxy-terminal half of apo-B100 necessary for clearance by the LDL receptor. Chylomicron triglyceride suffers the same fate as VLDL triglyceride and the chylomicron remnant circulates back to the liver, where its clearance is mediated by the interaction of apo-E with lipoprotein receptors.

Evidence for the existence of a distinct hepatic apo-E receptor comes from various sources ${ }^{1,2}$. Most convincingly, humans with familial hypercholesterolaemia and rabbits do not have elevated levels of chylomicron remnants or of other lipoproteins cleared by apo-E. Two lowmolecular-mass apo-E binding proteins

From Nature 39, 461; 14 March 1889.

It seems clear that the ledges owe their origin to the action of rain-water, which would naturally penetrate below the surface covering of grass, and dissolve portions of the porous soil below. The grass layer would eventually have nothing to support it in places, and would collapse to a lower level. The effect of collapse would necessarily be a wrinkle or ledge at right angles to the ground slope. Eimund J. MiLls. have been suggested as candidates for the apo-E receptor, but this role has not been shown definitively. Herz et al. ${ }^{2}$ have now isolated complementary DNA clones encoding a 4,544-residue membrane glycoprotein very similar to the LDL receptor which could be the apo-E receptor. The protein predicted from this DNA sequence is abundant in the liver, lung and brain, but the messenger RNA encoding it is found in most tissues.

The LDL receptor is an 839-aminoacid multidomain protein ${ }^{2.5}$. The amino terminus contains seven, 40-residue, cysteine-rich repeats, each with a cluster of acidic residues near its carboxy terminus. The first repeat binds calcium which is required for ligand-receptor interaction; the other six bind regions of apo$\mathrm{B} 100$ and apo-E that are enriched in basic residues.

Cysteine-rich repeats similar to those at the amino terminus of the LDL receptor are found in components of the complement complex which may stabilize cytolytic conformation of the complex by binding to domains enriched in basic residues on other proteins. Herz et al. ${ }^{2}$ argued that proteins like the LDL receptor that interact with basic residues on apo-B100 and apo-E should contain conserved regions enriched in acidic residues, and indeed they found a complementary DNA in a mouse lymphocyte library encoding a protein very similar to the $\mathrm{LDL}$ receptor, which they call LDL receptor-related protein (LRP). This protein contains four repeats of the extracellular portion of the LDL receptor containing the ligand binding site, a domain similar to the epidermal growth factor (EGF) precursor, as well as a transmembrane segment and a cytoplasmic tail with two copies of the signal used by the LDL receptor for clustering in clathrin-coated pits. Although it remains to be confirmed that the protein is a lipoprotein receptor, its resemblance to the LDL receptor, together with its antibodyand calcium-binding properties, suggest that it has this function. If LRP is the apo$E$ receptor, the finding that its $m R N A$ is widely expressed outside the liver implies a more general role, perhaps paralleling the involvement of apo- $E$ in the repair of injured nerves and in the modulation of immune responses and cell growth ${ }^{\prime}$

The EGF precursor, LDL receptor and LRP are all remarkably similar over a 400 amino-acid region ${ }^{2.5}$, indicating a close evolutionary relationship. In the LDL receptor, the EGF-like domain is required for acid-dependent dissociation of the ligand from the receptor in the endosome after endocytosis and for repeated recycling of the receptor to the cell surface ${ }^{6}$. This domain in LRP could have a similar role. There are six other EGF repeats not present in the LDL receptor adjacent to the plasma membrane in LRP. The EGF precursor contains a similar grouping, 